\title{
A synthetic approach to chiral carbocyclic nucleosides of varied ring-sizes using carbon framework of D-glucose
}

\author{
Kaushik Singha, Basudeb Achari, and Sukhendu B. Mandal* \\ Division of Medicinal Chemistry, Indian Institute of Chemical Biology, Jadavpur, \\ Kolkata 700 032, India \\ E-mail: sbmandal@iicb.res.in
}

\section{Dedicated to Professor (Mrs.) Asima Chatterjee on her $85^{\text {th }}$ birth anniversary}

(received 27 Nov 03; accepted 15 Feb 04; published on the web 28 Feb 04)

\begin{abstract}
Synthesis of enantiomerically pure carbocyclic nucleoside analogues 10-16 with five-, six- and seven- membered rings has been achieved starting from D-glucose derived tetracyclic isoxazolidinocarbocycle precursors 1-3. Cyclization of 6-chloro pyrimidine derivatives 7-9 to purine derivatives was found to be accomplished by nucleophilic displacement of 6-chloro substituent (by dimethylamino and/or methoxy groups). Apparently, hydrogen bonding between $\mathrm{N}-3$ of the purine ring and a hydoxy substituent at $\mathrm{C}-2^{\prime}$ plays a crucial role in this transformation.
\end{abstract}

Keywords: Synthesis, chiral, carbocyclic nucleosides, D-glucose

\section{Introduction}

After the discovery of the two natural carbocyclic nucleosides (-)- $\operatorname{aristeromycin}^{1}$ and (-)neplanocin $\mathrm{A}^{2}$ possessing antineoplastic activity, considerable attention was paid towards the synthesis of carbocyclic nucleosides with cyclopentane ring $^{3-4}$ over the last decade. But little effort has been paid towards the synthesis of such molecules with different ring sizes ${ }^{5}$, particularly with six- and seven-membered rings. Besides, the realization that the biological activity normally resides in a particular enantiomer has given an impetus to the task of generating enantiomerically pure carbocyclic nucleosides. For an easy access to this class of chiral nucleosides, the synthesis of appropriate aminocarbocycles/hydroxycarbocycles in optically pure form is desirable. Though cyclopentadiene is often used for asymmetric synthesis of chiral amino- or hydroxy-cyclopentane derivatives ${ }^{4}$, the other procedures to prepare these carbocycles involve (i) ring closing metathesis reaction between two olefins ${ }^{6}$, (ii) manipulative degradation of norbornadiene skeleton and construction of 5-membered ring $^{7}$, (iii) construction of cyclopentane ring system through Michael-Aldol cyclization of appropriate intermediates ${ }^{8}$ and 
(iv) preparation of requisite substrates from commercially available carbohydrates ${ }^{9}$. It is conceivable that carbohydrates could be ideal precursors for preparing chiral carbocyclic nucleosides. An additional advantage of this approach in using carbohydrates lies in the fact that the optically pure aminocarbocycles are occasionally utilized as potential glycosidase inhibitors and as antibiotics as well.

We had previously shown that chiral carbocycles of different ring sizes ${ }^{10}$ fused to furanose ring could be constructed through the application of intramolecular nitrone cycloaddition reaction on glucose-derived nitrones. As a part of our programme in search of analogues of newer chiral carbocyclic nucleosides, we wish to report herein the useful conversion of these furanocarbocycles towards the synthesis of such nucleosides with five-, six- and sevenmembered carbocyclic rings.

\section{Results and Discussion}

The successful synthesis of optically active functionalized carbocycles ${ }^{10}$ by the application of intramolecular nitrone cycloaddition reaction between C-5 nitrone and C-3 olefin (glucose numbering) prompted us to extend our work towards the synthesis of carbocyclic nucleosides 1016 as shown in Scheme 1. Thus, removal of 1, 2-O-isopropylidene groups of 1-3 under acidic conditions followed by vicinal diol cleavage ${ }^{11}$ with $\mathrm{NaIO}_{4}$ in aqueous $\mathrm{EtOH}$ and reduction with $\mathrm{NaBH}_{4}$ provided the corresponding isoxazolidinocarbocycle derivatives 4-6 in good yields (58$71 \%)$. Hydrogenolysis of 4-6 with $\mathrm{Pd} / \mathrm{C}(10 \%) /$ cyclohexene $^{12}$ cleaved the isoxazolidine rings to afford the respective crude trihydroxy aminocyclopentane derivatives, which without further purification were coupled with 5-amino-4, 6-dichloropyrimidine to provide diaminochloropyrimidine compounds 7-9 (75-80\%). Treatment of 7 with $\mathrm{HC}(\mathrm{OEt})_{3} / p-\mathrm{TSA}$ in DMF at a temperature of $20^{\circ} \mathrm{C}$ furnished the unexpected 6-dimethylamino-, and 6-methoxy-purine carbocyclic nucleosides 10 (25\%) and 11 (21\%). Under similar condition, 8 afforded 12 (24\%) and $13(20 \%)$, but 9 yielded 14 (19\%) and 15 (32\%). Ammonolysis of 14 in MeOH cleanly furnished 16 in good yield. 


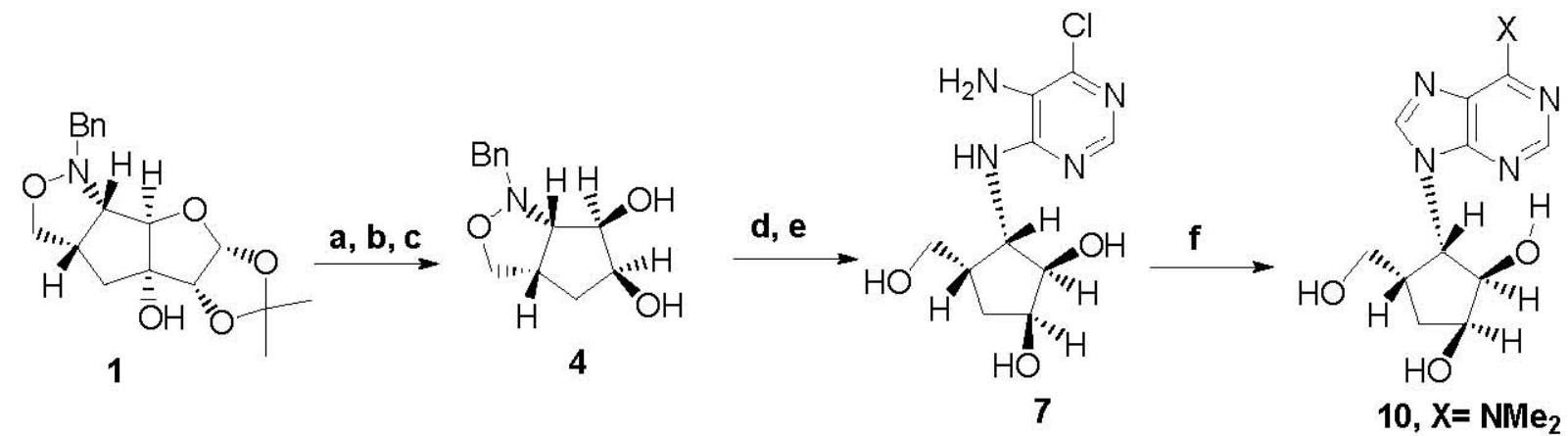

$11, X=O M e$

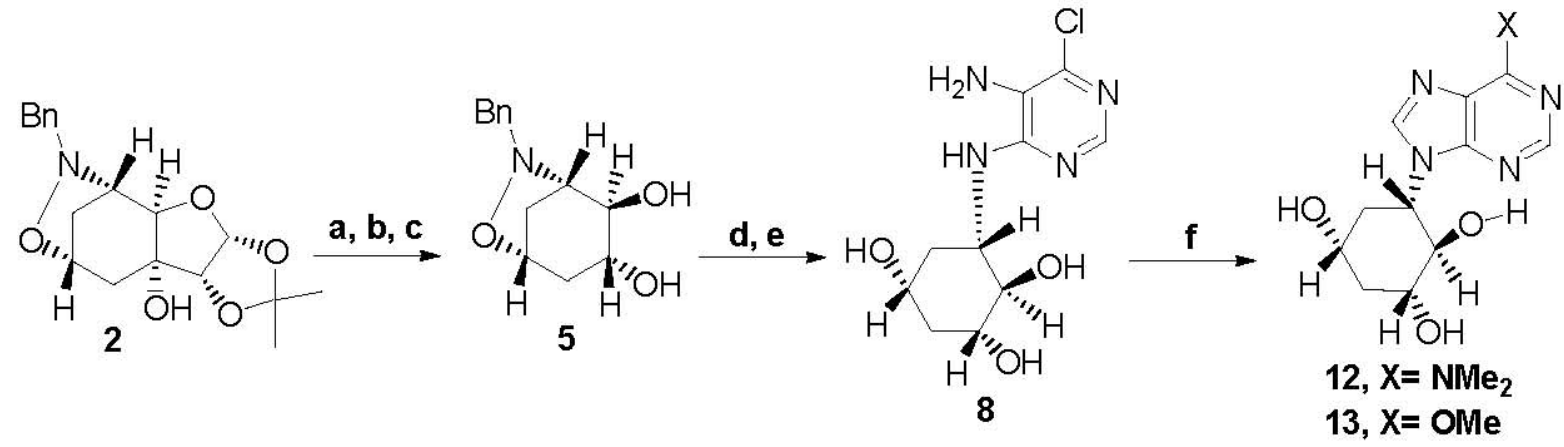

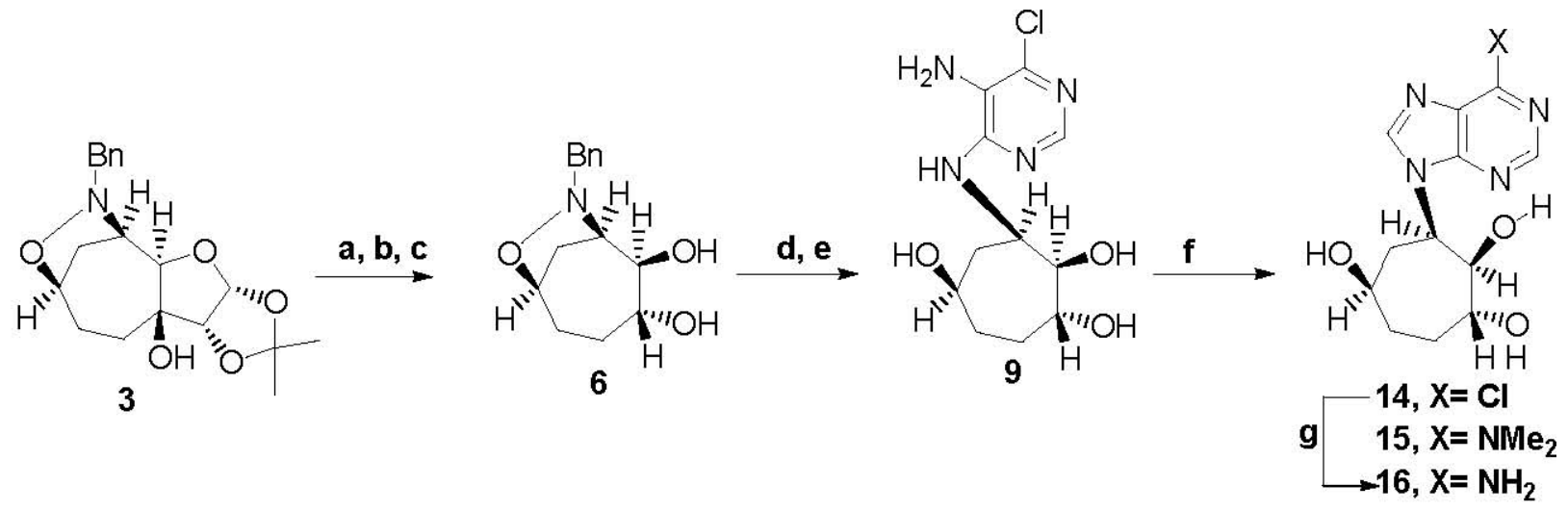

a Reagents: (a) $4 \% \mathrm{H}_{2} \mathrm{SO}_{4}, \mathrm{MeCN}, \mathrm{H}_{2} \mathrm{O}$, rt; (b) $\mathrm{NalO}_{4}$ (1.9 equiv), EtOH, $\mathrm{H}_{2} \mathrm{O}, \mathrm{O}^{\circ} \mathrm{C}$; (c) $\mathrm{NaBH}_{4}, \mathrm{MeOH}$, $10^{\circ} \mathrm{C}$; (d) $10 \% \mathrm{Pd} / \mathrm{C}$, cyclohexene, EtOH, reflux; (e) 5-amino-4, 6-dichloropyrimidine, $n$-BuOH, $\mathrm{Et}_{3} \mathrm{~N}$, reflux, (f) $\mathrm{HC}(\mathrm{OEt})_{3}, p-\mathrm{TSA}, \mathrm{DMF}, 20^{\circ} \mathrm{C}$; (g) $\mathrm{NH}_{3}, \mathrm{MeOH}, 80^{\circ} \mathrm{C}$, sealed tube

\section{Scheme 1}

In the cyclization reaction the presumed chloropurine derivatives generated in situ underwent facile transformation to the respective dimethylaminopurine nucleosides 10, 12 and 15 conceivably through nucleophilic displacement of the chloro group by dimethylamine derived from DMF. The formation of these products may be due to H-bonding between N-3 of purine 
ring and a hydroxy substituent at C-2/ facilitating nucleophilic attack at C-6 by nucleophiles. The minor products 11 and 13 were found to be methoxypurine analogues, which were formed during purification of nucleosides by reversed phase HPLC using $\mathrm{H}_{2} \mathrm{O}-\mathrm{MeOH}$ solvent system. The chloronucleoside 14 was, however, unchanged during chromatography and in this case no methoxypurine nucleoside was isolated.

To find out the possible role of the hydroxyl group adjacent to the amino group in influencing the ease of substitution of chloro group by $\mathrm{NMe}_{2}$ and methoxy groups, we wanted to protect the 2'-hydroxyl groups in $\mathbf{4}$ and $\mathbf{5}$. While $\mathbf{4}$ was easily diallylated to 17, attempted diallylation of the hydroxyl groups in 5 furnished only the monoallylated derivative 18 presumably due to steric hindrance offered by the bridged ring. The compounds $\mathbf{1 7}$ and $\mathbf{1 8}$ on transfer hydrogenolysis with cyclohexene-Pd/C treatment furnished crude di-n-propyl aminocyclopentane carbocycles, which on cyclization by the usual method furnished only the chloronucleoside analogues 20 and 21 (Scheme 2).

Removal of the acetonide functionality from each of the compounds 1-3 by acid treatment was evident from the disappearance of two distinctive methyl signals at $\delta \sim 1.30$ and 1.50. The generation of two hydroxyl groups in 4-6 was confirmed after their conversion into diacetates. In each case two acetoxy methyl peaks at $\sim \delta 2.1-2.2$ were observed in their ${ }^{1} \mathrm{H}$ NMR spectra. The trans-disposition of the 2,3- hydroxyl groups in $\mathbf{5}$ and $\mathbf{6}$ was indicated by the recovery of the starting diols from attempted periodate oxidation. However, the same periodate oxidation reaction on 4 resulted in the formation of a dialdehyde characterized by the peak at $1730 \mathrm{~cm}^{-1}$ in the IR spectrum of the crude product indicating the cleavage of the cyclopentane ring. During the reaction sequences, the isoxazolidine ring was not disturbed as was evident from the presence of aromatic and benzylic protons in the ${ }^{1} \mathrm{H}$ NMR spectra of the compounds. However, the absence of aromatic as well as benzylic proton signals in the ${ }^{1} \mathrm{H}$ NMR spectra of the crude products obtained by hydrogenolysis of 4-6 suggested the cleavage of the isoxazolidine rings accompanied by debenzylation.

Formation of the products 7-9 from the coupling reactions of the aminocarbocycles with 5amino-4, 6-dichloropyrimidine was evident from the appearance of a one-proton singlet at $\delta \sim 7.8$ in the ${ }^{1} \mathrm{H}$ NMR spectrum which is characteristic for the aromatic $\mathrm{H}-2$. On cyclization, the two aromatic proton singlets appeared at $\delta \sim 8.1-8.5$ in all the purine nucleosides. The characteristic feature in the ${ }^{1} \mathrm{H}$ NMR spectrum (in DMSO- $d_{6}$ ) of the compounds 10, 12 and 15 was the presence of a very broad signal at $\delta \sim 3.45$ assigned to $\mathrm{NMe}_{2}$. At $60^{\circ} \mathrm{C}$ the broad peak changed to a sharp singlet. Additionally, the ${ }^{13} \mathrm{C}$ signal for $\mathrm{NMe}_{2}$ of these compounds (in DMSO- $d_{6}$ ) could not be detected at normal temperature but appeared as a sharp singlet at $\delta \sim 38.8$ at an elevated temperature $\left(60^{0} \mathrm{C}\right)$. The ${ }^{1} \mathrm{H}$ NMR spectrum of $\mathbf{1 1}$ and $\mathbf{1 3}$ exhibited a sharp singlet at $\delta .4 .10$, characteristic for the $\mathrm{OCH}_{3}$, in addition to the aromatic proton signals at $\delta 8.39$ and 8.51 (for 11) and 8.36 and 8.50 (for 13). The presence of ${ }^{13} \mathrm{C}$ peaks at $\delta \sim 53.8$ (OMe), 143.3, 151.0 (aromatic $\mathrm{CH})$ in addition to other appropriate signals are also in conformity with the assigned structures. The mass spectra (FABMS) of 14 and 16 exhibited molecular ion peaks at $m / z 299$ and 301 (for 14) and at 280 (for 16). 
The ${ }^{1} \mathrm{H}$ NMR signals of the two methylene groups of the compound $\mathbf{1 8}$ appeared at $\delta 1.74$, 2.06 and 2.36; $\mathrm{N}-\mathrm{CH}$ (isoxazolidine ring juncture) as well as $\mathrm{CH}-\mathrm{O}$ allyl signals were found at $\delta$ 3.61, $\mathrm{CH}-\mathrm{O}$ (isoxazolidine ring juncture) signal at $\delta 4.58$ and $\mathrm{CH}-\mathrm{OH}$ signal at $\delta 4.65$. In confirmation, the proton signal at $\delta 4.65$ shifted downfield to $\delta 4.85$ after acetylation. Finally, upon irradiation of the signal at $\delta 4.85$ of the acetylated product 19, the doublet of a double doublet (ddd) at $\delta 2.02(J=15,7,2.5 \mathrm{~Hz})$ for one of the methylene protons changed to a broad doublet $(J=16 \mathrm{~Hz})$. Similarly, upon irradiation of the peak at $\delta 4.58$, the same methylene signal appeared as dd $(J=7,15 \mathrm{~Hz})$, confirming the substitution pattern.
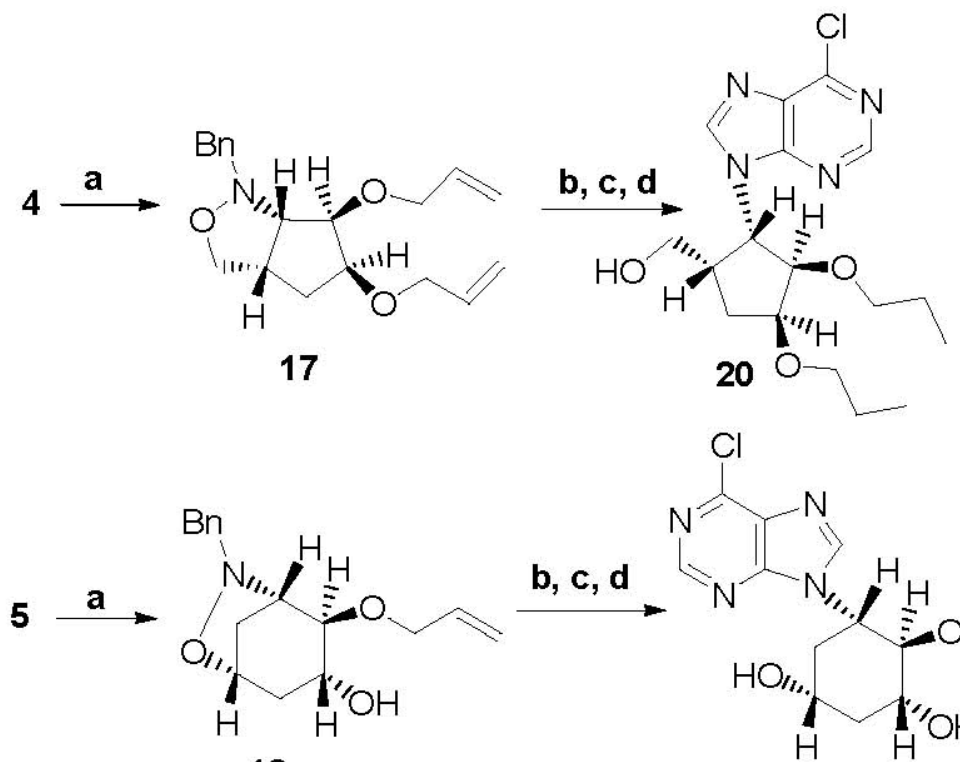

18
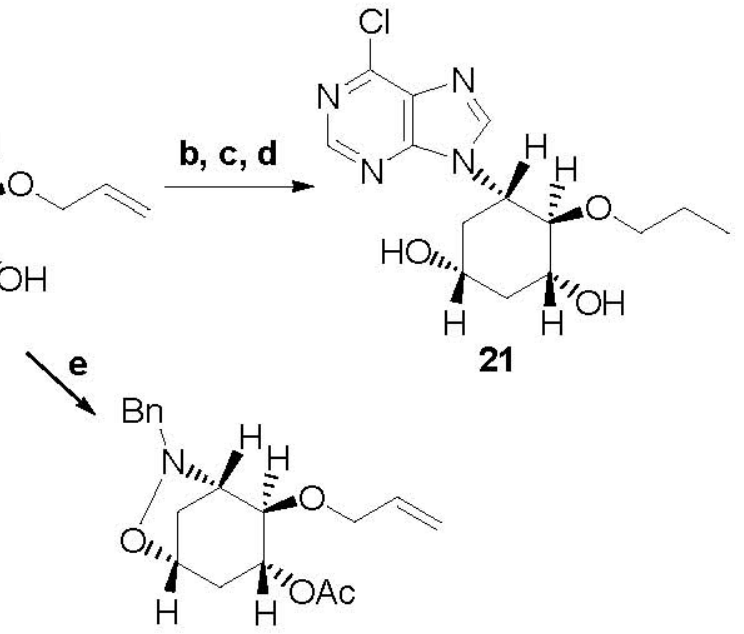

21

19

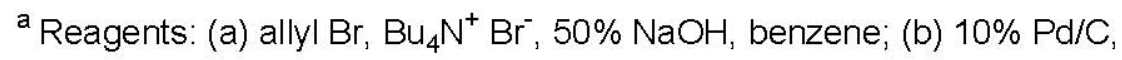

cyclohexene, EtOH, reflux, $\mathrm{N}_{2}$; (c) 5-amino-4, 6-dichloropyrimidine, n-BuOH,

$\mathrm{Et}_{3} \mathrm{~N}$, reflux; (d) $\mathrm{HC}(\mathrm{OEt})_{3}, \mathrm{p}-\mathrm{TSA}, \mathrm{DMF}, 2 \mathrm{O}^{\circ} \mathrm{C}$; (e) $\mathrm{Ac}_{2} \mathrm{O}, \mathrm{py}, \mathrm{rt}, 12 \mathrm{~h}$

\section{Scheme 2}

The ${ }^{1} \mathrm{H}$ NMR spectrum of $\mathbf{2 0}$ did not show any signal for $\mathrm{NMe}_{2}$ group and the FABMS showed the molecular ion peak at $\mathrm{m} / \mathrm{z} 369\left(\mathrm{MH}^{+}\right.$for $\left.\mathrm{Cl}^{35}\right)$ and $371\left(\mathrm{MH}^{+}\right.$for $\left.\mathrm{Cl}^{37}\right)$. Similar results were obtained with the nucleoside 21 (protonated molecular ion peaks at $\mathrm{m} / \mathrm{z} 327$ and 329). Thus, the participation of $2 /-\mathrm{OH}$ group in generating dimethylaminopurine and methoxypurine 
nucleoside analogues through nucleophilic displacement of chloro group is obvious.

In conclusion, the present work mainly deals with an efficient synthetic route to enantiomerically pure carbocyclic nucleoside analogues with five-, six- and seven-membered rings starting from D-glucose derived precursors. The neighbouring 2 - $-\mathrm{OH}$ group is involved in forming hydrogen bond with $\mathrm{N}-3$ of purine nucleus and helps to displace C- 6 chloro group by nucleophiles like dimethyl amino and methoxy groups.

\section{Experimental Section}

General Procedures. Melting points were taken in open capillaries and are uncorrected. IR spectra were measured on a JASCO 700 spectrophotometer. ${ }^{1} \mathrm{H}$ and ${ }^{13} \mathrm{C}$ NMR spectra were measured either on a JEOL FX-100 or on a Bruker AM 300 L spectrometer using TMS as internal standard. Mass spectra were obtained using a JEOL AX-500 spectrometer operating at $70 \mathrm{eV}$. Optical rotations were measured in a JASCO DIP 360 polarimeter. HPLC was performed on $\mu$ Bondapak ${ }^{\mathrm{TM}} \mathrm{C}_{18}$ column $(7.8 \times 300 \mathrm{~mm})$. Flash chromatography was carried out on LiChroprep ${ }^{\mathrm{R}}$ RP-18 (Merck).

\section{Preparation of (3aS, 5S, 6R, 6aR)-1-benzyl-hexahydrocyclopenta[c] isoxazole-5, 6-diol (4).} Compound $1(500 \mathrm{mg}, 1.5 \mathrm{mmol})$ was stirred with $4 \% \mathrm{H}_{2} \mathrm{SO}_{4}$ in $\mathrm{CH}_{3} \mathrm{CN}-\mathrm{H}_{2} \mathrm{O}(3: 1,30 \mathrm{~mL})$ for $24 \mathrm{~h}$ at $\mathrm{rt}$. After the completion of the reaction, the solution was neutralized with solid $\mathrm{CaCO}_{3}$, filtered, dried over anhydrous $\mathrm{Na}_{2} \mathrm{SO}_{4}$ and evaporated to a thick colourless liquid (400mg). This was dissolved in EtOH $(25 \mathrm{~mL})$ and cooled to $0^{\circ} \mathrm{C}$. An aqueous solution $(25 \mathrm{~mL})$ of $\mathrm{NaIO}_{4}(555$ $\mathrm{mg}, 2.6 \mathrm{mmol}, 1.9$ equiv) was added to this solution dropwise with vigorous stirring. After 40 min of stirring, the mixture was filtered, the filtrate was evaporated, and the residue was dissolved in $\mathrm{CHCl}_{3}(100 \mathrm{~mL})$. The $\mathrm{CHCl}_{3}$ solution was washed with $\mathrm{H}_{2} \mathrm{O}(2 \times 40 \mathrm{~mL})$ and dried $\left(\mathrm{Na}_{2} \mathrm{SO}_{4}\right)$; evaporation of the solvent afforded a crude ketone (IR $\left.1770 \mathrm{~cm}^{-1}\right)$.

To this ketone dissolved in $\mathrm{MeOH}(40 \mathrm{~mL})$ at $10^{\circ} \mathrm{C}$ was added $\mathrm{NaBH}_{4}(2 \times 125 \mathrm{mg})$ portionwise and the mixture was stirred for $5 \mathrm{~h}$. The solvent was evaporated, brine $(20 \mathrm{~mL})$ was added to the residue, and the crude product was extracted with $\mathrm{CHCl}_{3}(2 \times 50 \mathrm{~mL})$. The combined $\mathrm{CHCl}_{3}$ extract was washed with $\mathrm{H}_{2} \mathrm{O}(2 \times 25 \mathrm{~mL})$, dried with anhydrous $\mathrm{Na}_{2} \mathrm{SO}_{4}$ and evaporated to give a residue, which was purified by column chromatography, eluting with $\mathrm{CHCl}_{3}-\mathrm{MeOH}$ (98:2) mixture to afford 4 (257 mg, 71\%): $\mathrm{mp} 127-128^{\circ} \mathrm{C} ;{ }^{1} \mathrm{H}$ NMR $\left(\mathrm{CDCl}_{3}, 300 \mathrm{MHz}\right): \delta 1.62$ (m, 1H), $2.29(\mathrm{~m}, 1 \mathrm{H}), 2.42$ (br signal, 1H), $3.14(\mathrm{~m}, 1 \mathrm{H}), 3.34$ (brdd, 1H), 3.67 (brdd, 1H), 3.87 $(\mathrm{m}, 3 \mathrm{H}), 4.02(\mathrm{~d}, 1 \mathrm{H}, J=13.0 \mathrm{~Hz}), 4.15(\mathrm{t}, 1 \mathrm{H}, J=8.0 \mathrm{~Hz}), 7.26-7.35(\mathrm{~m}, 5 \mathrm{H}) ;{ }^{13} \mathrm{C} \mathrm{NMR}\left(\mathrm{CDCl}_{3}\right.$, $75 \mathrm{MHz}$ ): $\delta 36.9,43.1,60.4,72.7,75.9,77.3,80.2,127.7,128.5$ (2C), 129.1(2C), 136.5; EIMS, m/z: $235\left(\mathrm{M}^{+}\right)$. Anal. Calcd for $\mathrm{C}_{13} \mathrm{H}_{17} \mathrm{NO}_{3}$ : C, 66.36; H, 7.28; N, 5.95 Found: C, 65.98; H, 7.20; N, 5.13 .

Preparation of (1S, 2R, 3R, 5R)- 7-benzyl-6-oxa-7-aza-bicyclo [3.2.1] octane-2, 3-diol (5).

The compound 5 (in $64 \%$ yield) was prepared following the method as used in the preparation of 
4 using the same protocol.

5. $\mathrm{mp} 131-133^{\circ} \mathrm{C} ;[\alpha]_{\mathrm{D}}{ }^{20}-138.0^{\circ}(\mathrm{c} 0.23, \mathrm{MeOH}) ;{ }^{1} \mathrm{H}$ NMR $\left(\mathrm{CDCl}_{3}, 300 \mathrm{MHz}\right): \delta 1.78(\mathrm{dd}, 1 \mathrm{H}$, $J=14.7,5.3 \mathrm{~Hz}), 1.97-2.08(\mathrm{~m}, 2 \mathrm{H}), 2.35$ (brs, $1 \mathrm{H}), 2.40(\mathrm{~d}, 1 \mathrm{H}, J=11.6 \mathrm{~Hz}), 3.57$ (t, $1 \mathrm{H}, J=5$ Hz), 3.62 (brd, 1H), $3.76(\mathrm{~d}, 1 \mathrm{H}, J=13.0 \mathrm{~Hz}), 3.95$ (brd, 1H), 4.13 (d, 1H, J = 13.0 Hz), 4.66 (t, $1 \mathrm{H}, J=5.0 \mathrm{~Hz}), 7.32(\mathrm{~m}, 5 \mathrm{H}) ;{ }^{13} \mathrm{C} \mathrm{NMR}\left(\mathrm{CDCl}_{3}, 75 \mathrm{MHz}\right): \delta 29.8,35.8,62.9,64.1,71.8,71.9$, 76.6, 127.6, 128.5 (2C), 128.9 (2C), 136.8; EIMS, m/z: $235\left(\mathrm{M}^{+}\right)$.

Anal. Calcd for $\mathrm{C}_{13} \mathrm{H}_{17} \mathrm{NO}_{3}$ : C, 66.36; H, 7.28; N, 5.95 Found: C, 66.18; H, 7.25; N, 5.92.

(1R, 2R, 3R, 5R)-8-Benzyl-5, 7-(epoxyimino) cycloheptane-1, 2-diol (6). Compound 3 (1.25 g, $3.36 \mathrm{mmol})$ was converted to the isoxazolidinocycloheptanediol $6(520 \mathrm{mg}, 58 \%)$ according to the method described in the preparation of 4.

6. thick oil; $[\alpha]^{25}+36.7^{\circ}(c 0.36, \mathrm{MeOH}) ;{ }^{1} \mathrm{H} \mathrm{NMR}\left(\mathrm{CDCl}_{3}+\mathrm{D}_{2} \mathrm{O}, 100 \mathrm{MHz}\right)$ : $\delta$ 1.48-2.20 (2xm, $5 \mathrm{H}), 2.24-2.64(\mathrm{~m}, 1 \mathrm{H}), 3.32-4.12(\mathrm{~m}, 7 \mathrm{H}$, consisting of $2 \mathrm{xd}, 1 \mathrm{H}$ each, $\delta 3.74$ and $4.00, J=13$ Hz each; t, 1H, $\delta 3.40, J=4 \mathrm{~Hz}$; dd, $1 \mathrm{H}, \delta 3.55, J=4,8 \mathrm{~Hz} ; \mathrm{m}, 1 \mathrm{H}, \delta 3.75$ ), 4.60 (brd, $1 \mathrm{H}), 7.35$ $(\mathrm{m}, 5 \mathrm{H}) ;{ }^{13} \mathrm{C} \mathrm{NMR}\left(\mathrm{CDCl}_{3}, 25 \mathrm{MHz}\right): \delta 24.9,28.4,29.8,63.1,67.1,71.6,72.0,76.6,127.1$, 128.0, 128.5, 136.6; FABMS, $\mathrm{m} / \mathrm{z}: 250\left(\mathrm{M}^{+}+1\right)$. Anal. Calcd for $\mathrm{C}_{14} \mathrm{H}_{19} \mathrm{NO}_{3}: \mathrm{C}, 67.45 ; \mathrm{H}, 7.68$; N, 5.62. Found: C, 67.45; H, 7.65; N, 5.25.

Preparation of $(1 S, \quad 2 R, \quad 3 S, \quad 4 S)$-3-(5-amino-6-chloro-pyrimidin-4-ylamino)-4hydroxymethyl-cyclopentane-1, 2-diol (7); (1R, 2R, 4R, 6S)-6-(5-amino-6-chloro-pyrimidin4-ylamino)-cyclohexane-1, 2, 4-triol (8) and $(1 R, 2 R, 3 R, 5 R)-3-(5$-amino-6-chloropyrimidin-4-ylamino)-cycloheptane-1,2,5-triol (9). The compound 4 (250 $\mathrm{mg}, 1.06 \mathrm{mmol}$, dried over $\left.\mathrm{P}_{2} \mathrm{O}_{5}\right)$ was dissolved in dry EtOH $(30 \mathrm{~mL})$ and heated at reflux after addition of $\mathrm{Pd} / \mathrm{C}$ $(10 \%, 150 \mathrm{mg})$ and cyclohexene $(3 \mathrm{~mL})$ for $5 \mathrm{~h}$ under $\mathrm{N}_{2}$. The reaction mixture was filtered and evaporated to get a residue, which was dried over $\mathrm{P}_{2} \mathrm{O}_{5}$. To the residue $(150 \mathrm{mg}, 1.02 \mathrm{mmol})$ dissolved in dry $n$ - $\mathrm{BuOH}(25 \mathrm{~mL})$ were added 5-amino- 4, 6-dichloro pyrimidine $(250 \mathrm{mg}, 1.53$ mmol, 1.5 eqv.) and $\mathrm{Et}_{3} \mathrm{~N}(2 \mathrm{~mL})$. The mixture was then heated at reflux for $20 \mathrm{~h}$ under $\mathrm{N}_{2}$ atmosphere. The solvent was evaporated off under reduced pressure to give a solid residue, the aqueous solution of which was washed with chloroform $(3 \times 10 \mathrm{~mL})$. The $\mathrm{CHCl}_{3}$ solution was dried $\left(\mathrm{Na}_{2} \mathrm{SO}_{4}\right)$ and evaporated to yield a yellowish solid, which was purified by column chromatography (silica gel). Elution with $\mathrm{CHCl}_{3}-\mathrm{MeOH}$ (93:7) furnished a pale yellow foamy solid identified as 7 (225 mg, 80\%): mp $123-124^{\circ} \mathrm{C}$; $[\alpha]_{\mathrm{D}}{ }^{20}-42.3^{\circ}$ (c 0.23, MeOH); ${ }^{1} \mathrm{H} \mathrm{NMR}$ $\left(\mathrm{D}_{2} \mathrm{O}, 300 \mathrm{MHz}\right): \delta 1.50(\mathrm{~m}, 1 \mathrm{H}), 2.28(\mathrm{~m}, 1 \mathrm{H}), 2.53(\mathrm{~m}, 1 \mathrm{H}), 3.43(\mathrm{dd}, 1 \mathrm{H}, J=11.4,6.5 \mathrm{~Hz})$, $3.52(\mathrm{dd}, 1 \mathrm{H}, J=11.4,5.4 \mathrm{~Hz}), 4.00(\mathrm{~m}, 2 \mathrm{H}), 4.30(\mathrm{t}, 1 \mathrm{H}, J=8.8 \mathrm{~Hz}), 7.80(\mathrm{~s}, 1 \mathrm{H}) ;{ }^{13} \mathrm{C}$ NMR $\left(\mathrm{D}_{2} \mathrm{O}, 75 \mathrm{MHz}\right): \delta 31.4,36.0,56.2,61.4,73.6,80.0,122.4,139.2,147.3,153.7 ; \mathrm{FABMS}, \mathrm{m} / \mathrm{z}$ : $275\left(\mathrm{M}^{+}+1\right.$, for $\left.\mathrm{Cl}^{35}\right), 277\left(\mathrm{M}^{+}+1\right.$, for $\left.\mathrm{Cl}^{37}\right)$.

Anal. Calcd for $\mathrm{C}_{10} \mathrm{H}_{15} \mathrm{ClN}_{4} \mathrm{O}_{3}$ : C, 43.72; H, 5.50;N, 20.40. Found: C, 43.12; H, 5.35; N, 19.95.

The compound 8 was prepared from 5 following the procedure as adopted in the preparation of 7 from 4 using the given protocol: 5 (215 mg, $0.91 \mathrm{mmol})$, dry EtOH $(30 \mathrm{~mL}), \mathrm{Pd} / \mathrm{C}(10 \%$, $150 \mathrm{mg})$ and cyclohexene $(3 \mathrm{~mL})$ for its conversion to a crude aminocarbocycle $(107 \mathrm{mg}, 0.72$ $\mathrm{mmol}$ ), then 5-amino- 4, 6-dichloro pyrimidine (177 mg, $1.10 \mathrm{mmol}, 1.5$ equiv.), $\mathrm{Et}_{3} \mathrm{~N}(2 \mathrm{~mL}), n$ $\mathrm{BuOH}(25 \mathrm{~mL})$ to prepare 8 (158 mg, 80\%): pale yellow foam; $[\alpha]_{\mathrm{D}}{ }^{20}-110.6^{\circ}(c 0.21, \mathrm{MeOH})$. 
${ }^{1} \mathrm{H}$ NMR (D $\left.\mathrm{D}_{2} \mathrm{O}, 300 \mathrm{MHz}\right): \delta 1.43(\mathrm{~m}, 2 \mathrm{H}), 2.27(\mathrm{~m}, 2 \mathrm{H}), 3.44$ (t-like, $\left.1 \mathrm{H}, J=9.5 \mathrm{~Hz}\right), 3.61(\mathrm{~m}$, 1H), $3.88(\mathrm{~m}, 1 \mathrm{H}), 4.03(\mathrm{~m}, 1 \mathrm{H}), 7.84(\mathrm{~s}, 1 \mathrm{H}) ;{ }^{13} \mathrm{C} \mathrm{NMR}\left(\mathrm{D}_{2} \mathrm{O}, 75 \mathrm{MHz}\right): \delta 38.1,39.9,50.5$, 64.2, 69.8, 76.6, 122.4, 139.5, 147.5, 153.5; FABMS, m/z: $275\left(\mathrm{M}^{+}+1\right.$, for $\left.\mathrm{Cl}^{35}\right), 277\left(\mathrm{M}^{+}+1\right.$, for $\mathrm{Cl}^{37}$ ). Anal. Calcd for $\mathrm{C}_{10} \mathrm{H}_{15} \mathrm{ClN}_{4} \mathrm{O}_{3}: \mathrm{C}, 43.72 ; \mathrm{H}, 5.50 ; \mathrm{N}, 20.40$. Found: $\mathrm{C}, 43.12 ; \mathrm{H}, 5.35 ; \mathrm{N}$, 19.95 .

The compound 9 was prepared from 6 following the procedure as described in the preparation of $\mathbf{8}$.

9. $[\alpha]_{\mathrm{D}}{ }^{20}+40.6^{\mathrm{o}}(\mathrm{c} 0.25, \mathrm{MeOH}) ;{ }^{1} \mathrm{H}$ NMR $\left(\mathrm{D}_{2} \mathrm{O}, 300 \mathrm{MHz}\right): \delta 1.43-2.25(\mathrm{~m}, 5 \mathrm{H}), 2.43(\mathrm{~m}, 1 \mathrm{H})$, 3.44 (t-like, $1 \mathrm{H}, J=9.5 \mathrm{~Hz}), 3.61(\mathrm{~m}, 1 \mathrm{H}), 3.88(\mathrm{~m}, 1 \mathrm{H}), 4.03(\mathrm{~m}, 1 \mathrm{H}), 7.84(\mathrm{~s}, 1 \mathrm{H}) ;{ }^{13} \mathrm{C}$ NMR $\left(\mathrm{D}_{2} \mathrm{O}, 75 \mathrm{MHz}\right): \delta 32.8,37.8,38.9,50.5,63.2,69.5,75.6,130.4,134.5,145.5,150.5$; FABMS, $\mathrm{m} / \mathrm{z}: 291\left(\mathrm{M}^{+}+1\right.$, for $\left.\mathrm{Cl}^{37}\right), 289\left(\mathrm{M}^{+}+1\right.$, for $\left.\mathrm{Cl}^{35}\right)$.

Preparation of $(1 S, 2 R, 3 S, 4 S)$-3-(6-dimethylamino-purin-9-yl)-4-hydroxymethylcyclopentane-1, 2-diol (10) and (1S, 2R, 3S, 4S)-4-hydroxymethyl-3-(6-methoxy-purin-9-yl)cyclopentane-1, 2-diol (11). To the compound 7 (210 mg, $0.76 \mathrm{mmol})$ dissolved in dry DMF (7 $\mathrm{mL})$ were added p-TSA (215 mg, $1.14 \mathrm{mmol})$ and freshly distilled $\mathrm{CH}(\mathrm{OEt})_{3}(2 \mathrm{~mL})$ and the reaction mixture was stirred for $24 \mathrm{~h}$ at room temperature under $\mathrm{N}_{2}$. The solvent DMF was evaporated under vacuum, the residue was dissolved in $\mathrm{MeOH}$ and the solution was treated with Dowex $\left(\mathrm{OH}^{-}\right)$for $5 \mathrm{~h}$. It was filtered and the filtrate was evaporated to give a solid material, which was subjected to flash chromatography using reverse phase material (LiChroprep) as stationary phase. TLC showed two overlapping compounds, which were finally separated by HPLC using isocratic solution of $\mathrm{H}_{2} \mathrm{O}-\mathrm{MeOH}(9: 1)$ to furnish the purine nucleoside analogues 10 (56 mg, $25 \%$ ) and $\mathbf{1 1}$ (44 mg, 21\%).

10. $\mathrm{mp} 138-139^{0} \mathrm{C} ;[\alpha]_{\mathrm{D}}{ }^{20}+65.7^{0}(\mathrm{c} 0.25, \mathrm{MeOH}) ;{ }^{1} \mathrm{H}$ NMR (DMSO-d $\left.6,300 \mathrm{MHz}\right): \delta 1.62(\mathrm{~m}$, $1 \mathrm{H}), 2.11(\mathrm{~m}, 1 \mathrm{H}), 2.45(\mathrm{~m}, 1 \mathrm{H}), 2.96\left(2 \mathrm{H}\right.$, brd, changing to $\mathrm{d}, J=5.7 \mathrm{~Hz}$ at $\left.60^{\circ} \mathrm{C}\right), 3.46(6 \mathrm{H}$, brs, changing to $\mathrm{s}$ at $\left.60^{\circ} \mathrm{C}\right), 3.87\left(1 \mathrm{H}\right.$, brq, changing to dd, $J=14.7,7.7 \mathrm{~Hz}$ on $\mathrm{D}_{2} \mathrm{O}$ exchange), $4.41\left(2 \mathrm{H}\right.$, brs, changing to $\mathrm{dd}, J=7.3,9.0 \mathrm{~Hz}$ on $\mathrm{D}_{2} \mathrm{O}$ exchange), $4.66(\mathrm{t}, 1 \mathrm{H}, J=9 \mathrm{~Hz}), 5.16$ (brs, 1H, exchangeable), 5.32 (brs, $1 \mathrm{H}$, exchangeable), $8.15(\mathrm{~s}, 1 \mathrm{H}), 8.20(\mathrm{~s}, 1 \mathrm{H})$; ${ }^{13} \mathrm{C} \mathrm{NMR}$ (DMSO-d 6 , 75 MHz): $\delta 32.9,37.7,59.9,60.7,74.3,78.5,119.0,139.2,150.9,151.4,154.1$, one signal merged inside DMSO signal; FABMS, m/z: $294\left(\mathrm{M}^{+}+1\right)$. Anal. Calcd for $\mathrm{C}_{13} \mathrm{H}_{19} \mathrm{~N}_{5} \mathrm{O}_{3}$ : C, 53.23; H, 6.53; N, 23.88. Found: C, 53.12; H, 6.4; N, 23.02.

11. $\mathrm{mp} 125-126^{0} \mathrm{C} ;[\alpha]_{\mathrm{D}}{ }^{20}+61.3^{0}$ (c $\left.0.25, \mathrm{MeOH}\right) ;{ }^{1} \mathrm{H}$ NMR (DMSO-d $\left.6,300 \mathrm{MHz}\right): \delta 1.59(\mathrm{~m}$, $1 \mathrm{H}), 2.10(\mathrm{~m}, 1 \mathrm{H}), 2.47(\mathrm{~m}, 1 \mathrm{H}), 3.00$ (brs, $2 \mathrm{H}), 3.87\left(1 \mathrm{H}\right.$, brq, changing to dd on $\mathrm{D}_{2} \mathrm{O}$ exchange), $4.10(\mathrm{~s}, 3 \mathrm{H}), 4.34$ (brs, $1 \mathrm{H}$, exchangeable), $4.46\left(1 \mathrm{H}\right.$, brt, changing to t-like on $\mathrm{D}_{2} \mathrm{O}$ exchange), 4.72 (t, 1H, $J=9.0 \mathrm{~Hz}$ ), 5.16 (brs, 1H, exchangeable), 5.35 (brs, 1H, exchangeable), $8.39(\mathrm{~s}, 1 \mathrm{H}), 8.51(\mathrm{~s}, 1 \mathrm{H}) ;{ }^{13} \mathrm{C} \mathrm{NMR}\left(\mathrm{DMSO}_{6}, \mathrm{~d}_{6}, 7 \mathrm{MHz}\right): \delta 32.9,37.6,53.7,60.4,60.8,74.0$, 78.5, 120.5, 143.4, 151.0, 152.9, 160.1; FABMS, m/z: $281\left(\mathrm{M}^{+}+1\right)$.

Anal. Calcd for $\mathrm{C}_{12} \mathrm{H}_{16} \mathrm{~N}_{4} \mathrm{O}_{4}$ : C, 51.42; H, 5.75; N, 19.99. Found: C, 51.12; H, 5.22; N, 19.11. Preparation of $(1 R, 2 R, 4 R, 6 S)$-6-(6-dimethylamino-purin-9-yl)-cyclohexane-1, 2, 4-triol (12) and (1R, 2R, 4R, 6S)- 6-(6-methoxy-purin-9-yl)-cyclohexane-1, 2, 4-triol (13). The compounds 12 and 13 were prepared from 8 following the method used in the preparation of the 
carbocyclic nucleoside derivatives $\mathbf{1 0}$ and $\mathbf{1 1}$ using the protocols: compound $\mathbf{8}(150 \mathrm{mg}, 0.55$ mmol), $p$-TSA (156 mg, $0.82 \mathrm{mmol}), \mathrm{CH}(\mathrm{OEt})_{3}(2 \mathrm{~mL})$, DMF (7 mL). Yield: 12 (40 mg, 24\%) and 13 (32 $\mathrm{mg}, 20 \%)$.

12. $\mathrm{mp} 253-255^{\circ} \mathrm{C} ;[\alpha]_{\mathrm{D}}{ }^{20}+15.4^{0}(c \mathrm{c} 0.24, \mathrm{MeOH}) ;{ }^{1} \mathrm{H}$ NMR (DMSO- $\left.\mathrm{d}_{6}, 300 \mathrm{MHz}\right): \delta 1.42$ (q, $1 \mathrm{H}, J=12.0 \mathrm{~Hz}), 2.06(\mathrm{~m}, 3 \mathrm{H}), 3.45\left(3 \mathrm{H}\right.$, brs, changing to $\mathrm{s}$ at $\left.60^{\circ} \mathrm{C}\right), 3.68(1 \mathrm{H}$, br signal, changing to $\mathrm{m}$ on $\mathrm{D}_{2} \mathrm{O}$ exchange), $3.82\left(1 \mathrm{H}\right.$, brt, changing to dd, $J=10,9 \mathrm{~Hz}$ on $\mathrm{D}_{2} \mathrm{O}$ exchange,), 4.21(m, 1H), 4.84, 4.89, 4.93 (3x brd, $1 \mathrm{H}$ each, exchangeable), $8.14(\mathrm{~s}, 1 \mathrm{H}), 8.18(\mathrm{~s}, 1 \mathrm{H}) ;{ }^{13} \mathrm{C}$ NMR (DMSO-d 6 , 75 MHz): $\delta 37.8,39.6,42.3,55.5,64.2,70.3,74.9,119.6,139.2,150.3,151.1$, 154.2; FABMS, m/z: $294\left(\mathrm{MH}^{+}\right)$.Anal. Calcd for $\mathrm{C}_{13} \mathrm{H}_{19} \mathrm{~N}_{5} \mathrm{O}_{3}: \mathrm{C}, 53.23 ; \mathrm{H}, 6.53 ; \mathrm{N}, 23.88$. Found: C, 53.00; H, 6.44; N, 23.99 .

13. $\mathrm{mp} 208-209^{0} \mathrm{C} ;[\alpha]_{\mathrm{D}}{ }^{20}+17.4^{0}(c \quad 0.22, \mathrm{MeOH}) ;{ }^{1} \mathrm{H}$ NMR (DMSO- $\left.\mathrm{d}_{6}, 300 \mathrm{MHz}\right): \delta 1.44$ (q, $1 \mathrm{H}, J=12 \mathrm{~Hz}), 2.07(\mathrm{~m}, 2 \mathrm{H}), 2.15(\mathrm{q}, 1 \mathrm{H}, J=12 \mathrm{~Hz}), 3.70(\mathrm{~m}, 1 \mathrm{H}), 3.82(1 \mathrm{H}, \mathrm{m}$, changing on $\mathrm{D}_{2} \mathrm{O}$ exchange to t-like, $\left.J=10 \mathrm{~Hz}\right), 4.09(\mathrm{~s}, 1 \mathrm{H}), 4.31(\mathrm{~m}, 1 \mathrm{H}), 4.88(\mathrm{~m}, 1 \mathrm{H}$, exchangeable), 5.00 (brd, exchangeable), $8.36(\mathrm{~s}, 1 \mathrm{H}), 8.50(\mathrm{~s}, 1 \mathrm{H}) ;{ }^{13} \mathrm{C}$ NMR (DMSO-d $\left.6,75 \mathrm{MHz}\right): \delta 40.3,42.3$, 53.7, 56.1, 64.2, 70.1, 75.1, 120.9, 143.3, 150.8, 152.1, 160.1; FABMS, m/z: $281\left(\mathrm{M}^{+}+1\right)$.

Anal. Calcd for $\mathrm{C}_{12} \mathrm{H}_{16} \mathrm{~N}_{4} \mathrm{O}_{4}$ : C, 51.42; H, 5.75; N, 19.99. Found: C, 51.09; H, 5.65; N, 18.32 .

Preparation of (1R, 2R, 3R, 5R)- 3-(6-chloro-purin-9-yl)-cycloheptane-1, 2, 5-triol (14), (1R, $2 R, 3 R, 5 R)$-3-(6-dimethylamino-purin-9-yl)-cycloheptane-1, 2, 5-triol (15) and (1R, 2R, 3R, 5R)- 3-(6-amino-purin-9-yl)-cycloheptane-1, 2, 5-triol (16). The compounds 14-16 were prepared from 9 following the same procedure used in the cyclization reaction of 7 using the protocols: 9 (200 mg, $0.7 \mathrm{mmol}), p$-TSA $(172 \mathrm{mg}, 1 \mathrm{mmol}), \mathrm{HC}(\mathrm{OEt})_{3}(3 \mathrm{~mL})$ and DMF (10 $\mathrm{mL})$. Yield: 14 (40 mg, $19 \%$ ) and 15 (70 mg, $32 \%)$. The chloro nucleoside 14 (10 mg) was heated in a sealed tube in $\mathrm{MeOH}$ saturated with ammonia $(5 \mathrm{~mL})$ for $40 \mathrm{~h}$. the tube was cooled, opened up, the solvent was evaporated and the crude product was purified by HPLC.

14. $\mathrm{mp} 184-186^{\circ} \mathrm{C} ;[\alpha]_{\mathrm{D}}{ }^{20}+49.0^{0}$ (c $0.20, \mathrm{MeOH}$ ); ${ }^{1} \mathrm{H}$ NMR (DMSO- $d_{6}, 300 \mathrm{MHz}$ ): $\delta 1.50-1.95$ $(\mathrm{m}, 5 \mathrm{H}), 2.58(1 \mathrm{H}$, merged with solvent peak), $3.71(\mathrm{brs}, 1 \mathrm{H}), 3.85(\mathrm{~m}, 2 \mathrm{H}), 4.75(\mathrm{~m}, 3 \mathrm{H}), 5.23$ $(\mathrm{s}, 1 \mathrm{H}), 8.68(\mathrm{~s}, 1 \mathrm{H}), 8.78(\mathrm{~s}, 1 \mathrm{H}) ;{ }^{13} \mathrm{C}$ NMR (DMSO- $\left.d_{6}, 75 \mathrm{MHz}\right): \delta 25.0,32.0,36.1,54.2,66.4$, 71.0, 74.3, 127.2, 130.5, 146.3, 148.8, 151.2; FABMS, $m / z: 299\left(\mathrm{M}^{+}+1\right.$, for $\left.\mathrm{Cl}^{35}\right), 301\left(\mathrm{M}^{+}+1\right.$, for $\left.\mathrm{Cl}^{37}\right)$.

15. $\mathrm{mp} 201-202^{\circ} \mathrm{C} ;[\alpha]_{\mathrm{D}}{ }^{20}+44.0^{0}(c 0.3, \mathrm{MeOH}) ;{ }^{1} \mathrm{H}-\mathrm{NMR}$ (DMSO- $\left.d_{6}, 300 \mathrm{MHz}\right): \delta 1.48(\mathrm{~m}$, $1 \mathrm{H}), 1.75(\mathrm{~m}, 3 \mathrm{H}), 1.95(\mathrm{~m}, 1 \mathrm{H}), 2.49(1 \mathrm{H}$, merged with solvent peak), 3.44 (brs, $6 \mathrm{H}), 3.65(\mathrm{~m}$, 1H), 3.79 (brs, 1H), $3.86(\mathrm{~m}, 1 \mathrm{H}), 4.60-4.66(\mathrm{~m}, 2 \mathrm{H}), 4.69$ (d, 1H, J=4.0 Hz), 5.22 (d, 1H, J= $4.0 \mathrm{~Hz}$ ), $8.12(\mathrm{~s}, 1 \mathrm{H}), 8.21(\mathrm{~s}, 1 \mathrm{H}) ;{ }^{13} \mathrm{C}-\mathrm{NMR}$ (DMSO- $\left.d_{6}, 75 \mathrm{MHz}\right): \delta 25.9,32.8,37.4,38.7$, 53.8, 67.3, 72.3, 75.6, 119.7, 139.3, 150.3, 152.3, 155.1; FABMS, m/z: $308\left(\mathrm{M}^{+}+1\right)$.

16. $\mathrm{mp} 208-210^{\circ} \mathrm{C} ;[\alpha]_{\mathrm{D}}{ }^{20}+44.7^{0}(c 0.41, \mathrm{MeOH}) ;{ }^{1} \mathrm{H}$ NMR (DMSO- $\left.d_{6,}, 300 \mathrm{MHz}\right): \delta 1.49(\mathrm{~m}$, $1 \mathrm{H}), 1.75(\mathrm{~m}, 3 \mathrm{H}), 1.93(\mathrm{~m}, 1 \mathrm{H}), 2.50(1 \mathrm{H}$, merged signal), 3.65 (brd, $1 \mathrm{H}, J=9.0 \mathrm{~Hz}), 3.85(\mathrm{~m}$, $2 \mathrm{H}), 4.57-4.71(\mathrm{~m}, 3 \mathrm{H}), 5.25(\mathrm{~d}, 1 \mathrm{H}, J=4.2 \mathrm{~Hz}), 7.19(\mathrm{~s}, 2 \mathrm{H}), 8.11(\mathrm{~s}, 1 \mathrm{H}), 8.14(\mathrm{~s}, 1 \mathrm{H}) ;{ }^{13} \mathrm{C}$ NMR (DMSO-d 6 , 75 MHz): $\delta 25.9,32.7,37.4,53.8,67.3,72.3,75.7,119.1,140.4,149.5,152.9$, 156.8; FABMS, $m / z: 280\left(\mathrm{M}^{+}+1\right)$. 
Preparation of 3aS, 5S, 6R, 6aS- 5, 6-bis-allyloxy-1-benzyl-tetrahydro-cyclopent[c]isoxazole (17). Allyl bromide (4 ml) was added to a mixture of 4 (300 mg, $1.3 \mathrm{mmol}), 50 \% \mathrm{NaOH}(50$ $\mathrm{mL})$, tetra butyl ammonium bromide $(41.8 \mathrm{mg}, 0.13 \mathrm{mmol})$ in benzene $(50 \mathrm{~mL})$ and the mixture was heated at reflux with vigorous stirring for $3 \mathrm{~h}$. The organic layer was separated, washed with water $(3 \times 15 \mathrm{~mL})$, dried $\left(\mathrm{Na}_{2} \mathrm{SO}_{4}\right)$, and evaporated to get a reddish syrupy material, which was purified by column chromatography (silica gel) using pet ether-chloroform (3:7) to afford 17 as a colourless syrup (286 mg, 70\%): $[\alpha]_{\mathrm{D}}{ }^{20}+38.4^{0}\left(c 0.98, \mathrm{CHCl}_{3}\right) ;{ }^{1} \mathrm{H} \mathrm{NMR}\left(\mathrm{CDCl}_{3}, 300 \mathrm{MHz}\right) \delta$ $1.51(\mathrm{~m}, 1 \mathrm{H}), 2.30(\mathrm{~m}, 1 \mathrm{H}), 3.04(\mathrm{ddt}, 1 \mathrm{H}, J=16.0,8.8,2.6 \mathrm{~Hz}), 3.40(\mathrm{~m}, 1 \mathrm{H}), 3.63(\mathrm{dd}, 1 \mathrm{H}, J=$ 2.6, 8.6 Hz,), 3.69-3.73 (m, 2H), $3.74(\mathrm{~d}, 1 \mathrm{H}, J=12.8 \mathrm{~Hz}), 3.85$ (ddt, $1 \mathrm{H}, J=12.8,5.5,1.2 \mathrm{~Hz}$ ) $3.96-4.09$ (m, 4H), 4.01(d, 1H, $J=12.8 \mathrm{~Hz}) 5.08$ (ddd, 1H, $J=10.3,3.0,1.2 \mathrm{~Hz}), 5.12(\mathrm{dd}, 1 \mathrm{H}$, $J=3.2,1.6 \mathrm{~Hz}), 5.16(\mathrm{ddd}, 1 \mathrm{H}, J=7.0,3.0,1.5 \mathrm{~Hz}), 5.25(\mathrm{ddd}, 1 \mathrm{H}, J=17.0,3.3,1.5 \mathrm{~Hz}), 5.72-$ $5.96(\mathrm{~m}, 2 \mathrm{H}), 7.32(\mathrm{~m}, 5 \mathrm{H}) ;{ }^{13} \mathrm{C} \mathrm{NMR}\left(\mathrm{CDCl}_{3}, 75 \mathrm{MHz}\right): \delta 34.2,41.7,60.2,70.7,70.9,71.5$, 73.4, 82.4, 87.7, 116.4, 116.5, 127.5, 128.4 (2C), 129.3 (2C), 134.9, 135.2, 137.1; EIMS, m/z: $315\left(\mathrm{M}^{+}\right)$. Anal. Calcd for $\mathrm{C}_{19} \mathrm{H}_{25} \mathrm{NO}_{3}: \mathrm{C}, 72.35 ; \mathrm{H}, 7.99: \mathrm{N}, 4.44$. Found: $\mathrm{C}, 72.12 ; \mathrm{H}, 7.69 ; \mathrm{N}$, 4.27 .

Preparation of $(1 S, 2 R, 3 R, 5 R)$-2-allyloxy-7-benzyl-6-oxa-7-aza-bicyclo[3.2.1]octan-3-ol (18). Allylation of the compound $5(200 \mathrm{mg}, 0.85 \mathrm{mmol})$ was carried out as the method described in the preparation of $\mathbf{1 7}$ using allyl bromide $(4 \mathrm{ml}), 50 \% \mathrm{NaOH}$ solution $(50 \mathrm{~mL})$, and benzene $(50 \mathrm{~mL})$.

18. colourless syrup (160 mg, 68\%); ${ }^{1} \mathrm{H}$ NMR $\left(\mathrm{CDCl}_{3}, 300 \mathrm{MHz}\right): \delta 1.74(\mathrm{dd}, 1 \mathrm{H}, J=14.6,5.3$ $\mathrm{Hz}), 2.06(\mathrm{~m}, 2 \mathrm{H}), 2.36(\mathrm{~d}, 1 \mathrm{H}, J=11.6 \mathrm{~Hz}), 3.61(\mathrm{~m}, 2 \mathrm{H}), 3.78(\mathrm{~d}, 1 \mathrm{H}, J=12.8 \mathrm{~Hz}$, partially overlapped by another signal), $3.98(2 \mathrm{xddt}, 2 \mathrm{x} 1 \mathrm{H}, J=13.0,5.5,1.5 \mathrm{~Hz}), 4.12(\mathrm{~d}, 1 \mathrm{H}, J=12.8$ $\mathrm{Hz}), 4.58$ (brs, 1H), 4.65 (t, 1H, $J=5.0 \mathrm{~Hz}$ ), 5.12 (ddd, 1H, $J=10.5,2.7,1.0 \mathrm{~Hz}), 5.20$ (ddd, 1H, $J=17.2,3.2,1.6 \mathrm{~Hz}), 5.84(\mathrm{~m}, 1 \mathrm{H}), 7.30(\mathrm{~m}, 5 \mathrm{H}) ;{ }^{13} \mathrm{C} \mathrm{NMR}\left(\mathrm{CDCl}_{3}, 75 \mathrm{MHz}\right): \delta 30.4,36.3,62.9$ (2C), 68.5, 70.4, 76.4, 78.7, 116.9, 127.6, 128.5 (2C), 128.8 (2C), 134.5, and 136.8.

EIMS, m/z: $275\left(\mathrm{M}^{+}\right)$; Anal. Calcd for $\mathrm{C}_{16} \mathrm{H}_{21} \mathrm{NO}_{3} ; \mathrm{C}, 69.79 ; \mathrm{H}, 7.69 ; \mathrm{N}, 5.09 ; \mathrm{O}, 17.43$. Found: C, 69.71; H, 7.55; N, 4.97.

Preparation of $(1 S, 2 R, 3 R, 5 R)$-2-allyloxy-7-benzyl-6-oxa-7-azabicyclo[3.2.1]octan-3-yl acetate (19). Compound $18(12 \mathrm{mg}, 0.04 \mathrm{mmol})$ dissolved in pyridine (4 mL) was acetylated with acetic anhydride $(1 \mathrm{~mL})$ at $70^{\circ} \mathrm{C}$ for $12 \mathrm{~h}$. Pyridine was distilled off by azeotropic distillation with toluene and the residue was purified by chromatography using pet etherchloroform (1:1) to afford $19(6 \mathrm{mg})$ as a creamy solid: $[\alpha]_{\mathrm{D}}{ }^{20}-82.2^{\circ}\left(c 0.14, \mathrm{CHCl}_{3}\right) ;{ }^{1} \mathrm{H} \mathrm{NMR}$ $\left(\mathrm{CDCl}_{3}, 300 \mathrm{MHz}\right): \delta 1.78(\mathrm{brd}, 1 \mathrm{H}, J=15.0 \mathrm{~Hz}), 2.02(\mathrm{ddd}, 1 \mathrm{H}, J=15.0,7.0,2.5 \mathrm{~Hz}), 2.11-2.18$ (m, 5H including $\mathrm{COCH}_{3}$ signal), 3.45 (t-like, $\left.1 \mathrm{H}, J=3.5 \mathrm{~Hz}\right), 3.53$ (brs, $\left.1 \mathrm{H}\right), 3.76(\mathrm{~d}, 1 \mathrm{H}, J=$ $13.0 \mathrm{~Hz}), 4.00$ (2xdd, 2x 1H, $J=13.0,5.6 \mathrm{~Hz}), 4.12$ (d, 1H, J=13.0 Hz), $4.56(\mathrm{~m}, 1 \mathrm{H}), 4.85$ (m, $1 \mathrm{H}), 5.10(\mathrm{~d}, 1 \mathrm{H}, J=10.3 \mathrm{~Hz}), 5.17(\mathrm{dd}, 1 \mathrm{H}, J=17.0,1.4 \mathrm{~Hz}), 5.81(\mathrm{~m}, 1 \mathrm{H}), 7.31(\mathrm{~m}, 5 \mathrm{H})$; EIMS, $m / z: 317\left(\mathrm{M}^{+}\right)$.

Preparation of $(1 S, 2 S, 3 R, 4 S)$-2-(6-chloro-purin-9-yl)-3, 4-dipropoxy-cyclopentyl methanol (20). The nucleoside 20 was obtained from 17 according to the method described earlier. 
20. sticky solid (15 mg, $21 \%) ;[\alpha]_{\mathrm{D}}{ }^{20}+12.2^{0}$ (c $\left.0.18, \mathrm{MeOH}\right) ;{ }^{1} \mathrm{H}$ NMR $\left(\mathrm{CD}_{3} \mathrm{OD}, 300 \mathrm{MHz}\right)$ : $0.66(\mathrm{~m}, 6 \mathrm{H}), 1.24(\mathrm{~m}, 4 \mathrm{H}), 1.53(\mathrm{~m}, 1 \mathrm{H}), 2.25(\mathrm{~m}, 1 \mathrm{H}), 2.82(\mathrm{~m}, 1 \mathrm{H}), 3.45(\mathrm{~m}, 3 \mathrm{H}), 3.73(\mathrm{~m}$, $4 \mathrm{H}), 4.30(\mathrm{t}, 1 \mathrm{H}, J=9.0 \mathrm{~Hz}), 8.19(\mathrm{~s}, 1 \mathrm{H}), 8.20(\mathrm{~s}, 1 \mathrm{H})$; FABMS, $\mathrm{m} / \mathrm{z}: 369\left(\mathrm{MH}^{+}\right.$, for $\left.\mathrm{Cl}^{35}\right), 371$ $\left(\mathrm{MH}^{+}\right.$, for $\left.\mathrm{Cl}^{37}\right)$.

Preparation of $(1 R, 3 R, 4 R, 5 S)$ - 5-(6-chloro-purin-9-yl)-4-propoxy-cyclohexane-1, 3-diol (21). The compound 18 (160 mg, $0.58 \mathrm{mmol})$ was converted to 21 (20 $\mathrm{mg}, 11 \%$ in $3 \mathrm{steps})$ adopting the procedure described in the preparation of the nucleosides $\mathbf{1 0}$ and $\mathbf{1 1}$ from $\mathbf{4}$.

21. ${ }^{1} \mathrm{H}$ NMR (CD $\left.\mathrm{OD}, 300 \mathrm{MHz}\right): 0.68(\mathrm{t}, 3 \mathrm{H}), 1.38(\mathrm{~m}, 2 \mathrm{H}), 1.50(\mathrm{~m}, 1 \mathrm{H}), 1.70(\mathrm{~m}, 2 \mathrm{H}), 2.41$ $(\mathrm{m}, 1 \mathrm{H}), 3.57(\mathrm{~m}, 3 \mathrm{H}), 4.00(\mathrm{~m}, 1 \mathrm{H}), 4.45(\mathrm{t}, 1 \mathrm{H}, J=6.0 \mathrm{~Hz}), 8.18(\mathrm{~s}, 1 \mathrm{H}), 8.24(\mathrm{~s}, 1 \mathrm{H})$; FABMS, $\mathrm{m} / \mathrm{z}: 327\left(\mathrm{MH}^{+}\right.$, for $\left.\mathrm{Cl}^{35}\right), 329\left(\mathrm{MH}^{+}\right.$, for $\left.\mathrm{Cl}^{37}\right)$.

\section{Acknowledgements}

Financial support from DST (Govt. of India) given to S.B.M. is gratefully acknowledged.

\section{References}

1. Kusuka, T.; Yamamoto, H.; Shibata, M.; Muroi, M.; Kishi, T.1; Mizuno, K. J. Antibiot. 1968, $21,255$.

2. Yaginuma, S.; Tsujino, M.; Muto, N.; Otani, M.; Hiyashi, M.; Ishimura, F.; Fujita, T.; Watanabe, S.; Matsuda, T.; Watanabe, T.; Abe, J. Curr. Chemother Infect. Dis., Int. Congr. 1980, 2, 1558.

3. Agrofoglio, L.; Suhas, E.; Farese, A.; Condom, R.; Challand, S. R.; Earl, R. A.; Guedj, R. Tetrahedron 1994, 50, 10611.

4. Crimmins, M.T. Tetrahedron 1998, 54, 9229.

5. (a) Wang, J.; Busson, R.; Blaton, N.; Rozenski, J.; Herdewijn, P. J. Org. Chem. 1998, 63, 3051.(b) Barral, K.; Halfon, P.; Pepe, G.; Camplo, M. Tetrahedron Lett. 2002, 43, 81. (c) Konkel, M. J.; Vince, R. Tetrahedron 1996, 52, 799. (d) Panda, J.; Ghosh, S.; Ghosh, S.J. Chem. Soc., Perkin Trans I 2001, 3013. (e) Fernandez, F.; Lopez C.; Hergueta, A. R. Tetrahedron 1995, 51, 10317. (f) Lee, M. G.; Du, J. F.; Chun, M. W.; Chu C. K. J. Org. Chem. 1997, 62, 1991. (g) Csuk, R.; Eversmann L. Tetrahedron 1998, 54, 6445.

6. (a) Gillaizeau, I.; Charamon, S.; Agrofoglio, L. A. Tetrahedron Lett. 2001, 42, 8817. (b) Gurjar, M. K.; Maheshwar, K. J. Org. Chem. 2001, 66, 7552. (c) Crimmins, M. T.; King, B. W.; Zuercher, W. J.; Choy, A. L. J. Org. Chem. 2000, 65, 8499. (d) Hong, J. H.; Shim, M. J.; Ro, B.O.; Ko, O. H. J. Org. Chem. 2002, 67, 6837.

7. Zhao, Y.; Yang, T.; Lee, M.; Lee D.; Newton M. G.; Chu, C. K. J. Org. Chem. 1995, 60, 5236. 
8. Ono, M.; Nishimura, K.; Tsubouchi, H.; Nagaoka Y.; Tomoika, K. J. Org. Chem. 2001, 66, 8199.

1. 9. (a) Chun, B. K.; Song G. Y.; Chu, C. K. J. Org. Chem. 2001, 66, 4852. (b) Lee, M. G.; Du, J. F.; Chun, M. W.; Chu C. K. J. Org. Chem. 1997, 62, 1991. (c) Comin, M. J.; Rodriguez J. B. Tetrahedron 2000, 56, 4639. (d) Roy, A.; Patra, R.; Achari, B.; Mandal, S.B. Synlett 1997, 1237.(e) Patra, R.; Bar, N. C.; Roy, A.; Achari, B.; Ghoshal, N.; Mandal. S.B. Tetrahedron 1996, 52, 11265.

9. Brimacomb, J. S.; Rollins, A. J.; Thompson, S. W. Carbohydr. Res. 1973, 31, 108.

10. Collins, P. M.; Ashwood, M. S.; Eder, H.; Wright, S. H. B.; Kennedy, D.J. Tetrahedron Lett. 1990, 34, 3585. 